\title{
PRIMITIVE POINTS ON ELLIPTIC CURVES
}

\author{
BY S. LANG AND H. TROTTER 1
}

Communicated by Olga Taussky Todd, September 23, 1976

A well-known conjecture of Artin predicts the density of primes for which a given rational number is a primitive root (cf. the introduction to his collected works). Our purpose here is to formulate an analogous conjecture on elliptic curves $A$, say defined over the rationals for concreteness. Let $a$ be a rational point of infinite order. We ask for the density of those primes $p$ such that the group $\bar{A}\left(\mathbf{F}_{p}\right)$ of rational points $\bmod p$ is cyclic, generated by the reduction $\bar{a}$ of $a \bmod p$. We shall use the Galois extensions $K_{l}=\mathrm{Q}\left(A_{l}, l^{-1} a\right)$ analogous to the splitting fields of the equations $X^{l}-a=0$ when $a$ is in the multiplicative group. We may say that $a$ is primitive for such primes. We let $\langle a\rangle$ be the cyclic group generated by $a$.

The affine group, equal to the extension of the translation group $A_{l}$ by $G L_{2}(l)$, operates on $l^{-1} a$. For simplicity we fix an element $u_{0} \in l^{-1} a$. Then we may represent an element $\sigma$ in the affine group by a pair $(\gamma, \tau)$ with $\gamma \in$ $G L_{2}(l)$ and a translation $\tau \in A_{l}$, such that

$$
(\gamma, \tau) u=u_{0}+\gamma\left(u-u_{0}\right)+\tau .
$$

The Galois group $\mathrm{Gal}\left(K_{l} / \mathrm{Q}\left(A_{l}\right)\right)$ can be identified with a group of translations, subgroup of $A_{l}$, and is equal to $A_{l}$ for almost all $l$ by a theorem of Bashmakov [Ba]. If $\sigma=(\gamma, \tau)$ as above, we have

$$
\sigma u=u \quad \text { if and only if }(\gamma-1)\left(u_{0}-u\right)=\tau .
$$

Let $\Delta$ be the discriminant of the curve. We want to give a condition on the Frobenius element $\sigma_{p}=\left(\gamma_{p}, \tau_{p}\right)$ in $G_{l}$ when $p \nmid \Delta l$ in order that the index of $\langle\bar{a}\rangle$ in $\bar{A}\left(\mathbf{F}_{p}\right)$ is divisible by $l$. Note that $l$ divides the order of $\bar{A}\left(\mathbf{F}_{p}\right)$ if and only if $\gamma_{p}$ has eigenvalue 1 . Furthermore, $\bar{A}\left(\mathbf{F}_{p}\right)=\operatorname{Ker}\left(\gamma_{p}-1\right)$.

If $\gamma_{p}=1$ then the index of $\langle\bar{a}\rangle$ is divisible by $l$.

Suppose on the other hand that $\operatorname{Ker}\left(\gamma_{p}-1\right)$ is cyclic of order $l$. Then the index of $\langle\bar{a}\rangle$ is divisible by $l$ if and only if there exists $b \in \bar{A}$ with $l b=\bar{a}$ and $b$ is fixed by $\sigma_{p}$. Indeed, if $\bar{a}$ has period divisible by $l$, and the index is divisible by $l$, then $\bar{a}$ is divisible by $l$ in $\bar{A}\left(\mathbf{F}_{p}\right)$, otherwise $\bar{A}\left(\mathbf{F}_{p}\right)$ would contain $\mathbf{Z}(l)^{2}$. The converse is clear. If $\bar{a}$ has period not divisible by $l$ then $l b=\bar{a}$ for some $b$ in $\langle\bar{a}\rangle$, so the assertion is also clear in this case.

We see that the index of $\langle\bar{a}\rangle$ is divisible by $l$ if and only if $\sigma_{p}$ lies in the AMS (MOS) subject classifications (1970). Primary 12A75, $14 \mathrm{G} 25$.

${ }^{1}$ Both authors supported by NSF grants. 
set $S_{l}^{\prime}$ consisting of all elements $(\gamma, \tau)$ such that $\gamma$ has eigenvalue 1 , and either $\gamma=1$, or $\operatorname{Ker}(\gamma-1)$ is cyclic and $\tau \in \operatorname{Im}(\gamma-1)$.

Let $S_{l}=G_{l}-S_{l}^{\prime}$. For any set of primes $L$ we let $S_{L}=\Pi_{l \in L} S_{l}$. We let $K_{L}$ be the compositum of all fields $K_{l}$ with $l \in L$, and $G_{L}=\operatorname{Gal}\left(K_{L} / Q\right)$. We let $P_{L, S}(x)$ be the set of primes $p \leqslant x$ such that $p \nmid \Delta$ and the Frobenius element $\left(p, K_{l} / \mathrm{Q}\right) \in S_{l}$ for all $l \in L$ and $l \neq p$. If $L$ is finite, we have by Tchebotarev,

$$
\lim _{x \rightarrow \infty} \frac{\left|P_{L, S}(x)\right|}{\pi(x)}=\frac{\left|S_{L} \cap G_{L}\right|}{\left|G_{L}\right|}=\delta_{L}(S),
$$

It is easy to see that the limit $\delta(S)=\operatorname{Lim}_{L} \delta_{L}(S)$ exists (for $L$ increasing to include all primes). The conjecture states that the limit is equal to the density of primes for which $a$ is primitive.

There exists a finite set $M$ of primes such that for any $L$ containing $M$ we have

$$
\delta_{L}=\delta_{M} \prod_{l \in L-M} \delta_{l}, \quad \text { where } \quad \delta_{l}=1-\frac{\left|S_{l}^{\prime} \cap G_{l}\right|}{\left|G_{l}\right|}
$$

This allows for effective computation of the conjectured density. One finds in all cases that $\delta_{l}=1+O\left(1 / l^{2}\right)$, so that the product is absolutely convergent. When there is no complex multiplication, for instance, and $G_{l}$ is the full affine group (which occurs for almost all $l$ ), then one finds

$$
\delta_{l}=1-\frac{\left|S_{l}^{\prime}\right|}{\left|G_{l}\right|} \quad \text { where } \quad \frac{\left|S_{l}^{\prime}\right|}{\left|G_{l}\right|}=\frac{l^{3}-l-1}{l^{2}\left(l^{3}-l^{2}-l+1\right)} .
$$

We have computed numerical values for some "Serre curves" (cf. [LT]) whose Galois group of torsion points is of index 2 in the full product of all $G L_{2}\left(\mathrm{Z}_{l}\right)$, for instance the three curves

$$
y^{2}+y=x^{3}-x, \quad y^{2}+y=x^{3}+x^{2}, \quad y^{2}+x y+y=x^{3}-x^{2} .
$$

In each case the point of infinite order is the point $(0,0)$, and the predicted density comes within three decimals of 0.440 . Among the first 200 primes, the expected number is then 88 , and the actual numbers are $91,96,91$ respectively. For 180 primes (leaving out the first 20 ) the expected number is 79.2 , and the actual numbers are 79, 84, 78 respectively, a good fit. One can of course handle in a similar way the density of primes $p$ such that $\bar{A}\left(\mathbf{F}_{p}\right)$ is cyclic (forget about $a$ ).

We have also considered the more general problem dealing with a free subgroup $\Gamma$ of rational points, rather than an infinite cyclic one. Let $M$ be an integer $>1$. We wish to characterize the possibility that the image under reduction

$$
\Gamma \rightarrow \bar{A}\left(\mathbf{F}_{p}\right) / M \bar{A}\left(\mathbf{F}_{p}\right)
$$


is surjective by a condition on the Frobenius element $\sigma_{p}$. For simplicity, fix a section $\lambda: \Gamma \rightarrow M^{-1} \Gamma$ such that $M \lambda a=a$ for all $a \in \Gamma$. This corresponds to choosing $u_{0}$ when $\Gamma$ is infinite cyclic, and determines a homomorphism $\tau: \Gamma \rightarrow$ $A_{M}$ such that

$$
\sigma u=\gamma(u-\lambda u)+\lambda M u+\tau M u
$$

for $u \in M^{-1} \Gamma$. We can identify an element $\sigma$ of the affine group in this case with a pair $(\gamma, \tau)$. We see this time that $\sigma u=u$ if and only if $(\gamma-1)(u-\lambda M u)$ $=-\tau M u$. If we let $T_{p}$ be the kernel of the map

$$
\Gamma \rightarrow \bar{A}\left(\mathbf{F}_{p}\right) / M \bar{A}\left(\mathbf{F}_{p}\right),
$$

then $T_{p}=\tau_{p}^{-1}\left(\left(\gamma_{p}-1\right) A_{M}\right)$, where $\sigma_{p}=\left(\gamma_{p}, \tau_{p}\right)$ is the Frobenius element. Note that we have an equality of indices

$$
\left(\bar{A}\left(\mathbf{F}_{p}\right): M A\left(\mathbf{F}_{p}\right)\right)=\left|\left(\bar{A}\left(\mathbf{F}_{p}\right) \cap \bar{A}_{M}\right)\right|=\left|\operatorname{Ker}\left(\gamma_{p}-1\right)\right| .
$$

In particular, take $M=l$ prime. We find:

The index of $\bar{\Gamma}$ in $\bar{A}\left(\mathbf{F}_{p}\right)$ is prime to $l$ if and only if

$$
\operatorname{dim} \tau(\Gamma)-\operatorname{dim}(\tau(\Gamma) \cap \operatorname{Im}(\gamma-1))=\operatorname{dim} \operatorname{Ker}(\gamma-1) .
$$

We define the bad set $S_{l}^{\prime}$ to consist of those $\sigma=(\gamma, \tau)$ such that either $\operatorname{Ker}(\gamma-1)$ is cyclic and $\tau(\Gamma) \subset \operatorname{Im}(\gamma-1)$, or $\operatorname{Ker}(\gamma-1)=A_{l}$ and $\operatorname{rank} \tau(\Gamma)=$ 0 or 1 . Then we obtain:

The index of $\bar{\Gamma}$ in $\bar{A}\left(\mathbf{F}_{p}\right)$ is divisible by $l$ if and only if the Frobenius element $\sigma_{p}$ lies in $S_{l}^{\prime}($ for $p \nmid \Delta l)$.

The same type of limit as before yields the conjectured density of primes such that $\bar{\Gamma}=\bar{A}\left(\mathbf{F}_{p}\right)$, (say for which $\Gamma$ is primitive).

Trying to prove the conjecture from the Riemann hypothesis in line with Hooley's work for the Artin case [H] met difficulties having to do with the larger degrees, behaving like $>><l^{6}$ (or $l^{4}$ in the complex multiplication case) rather than $l^{2}$ in the Artin case. It also leads to the problem of proving the analogue of the Brun-Titchmarsh theorem, to given an upper bound for the number of primes $p \leqslant x$ such that the Frobenius at $p$ operating on $A_{l}$ has eigenvalue 1 .

\section{BIBLIOGRAPHY}

[Ba] M. I. Bašmakov, Un théorème de finitude sur la cohomologie des courbes élliptiques, C. R. Acad. Sci. Paris Sér. A-B 270 (1970), A999-A1101. MR 42 \#4548.

[Go] L. Goldstein, Analogues of Artin's conjecture, Trans. Amer. Math. Soc. 149 (1970), 431-442. MR 43 \#4792.

[Ho] C. Hooley, On Artin's conjecture, J. Reine Angew. Math. 225 (1967), 209220. MR 34 \#7445.

[LT] S. Lang and H. Trotter, Frobenius distributions in $G L_{2}$-extensions, Lecture Notes in Math., vol. 504, Springer-Verlag, Berlin and New York, 19.75. 
[We] P. Weinberger, A counterexample to an analogue of Artin's conjecture, Proc. Amer. Math. Soc. 35 (1972), 49-52. MR 45 \#8630.

DEPARTMENT OF MATHEMATICS, YALE UNIVERSITY, NEW HAVEN, CONNECTICUT 06520

DEPARTMENT OF MATHEMATICS, PRINCETON UNIVERSITY, PRINCETON, NEW JERSEY 08540 